\title{
How to transform care?
}

\author{
Trevor Jackson deputy editor, The BMJ
}

With just a few weeks to go to the UK general election, the two main political parties have been slugging it out over who will pay more for the NHS. In his weekly Election Watch column (doi:10.1136/bmj.h2009) The BMJ's news reporter, Gareth Iacobucci, unpicks the financial pledges made by Labour and the Conservatives. And in a one-off Observations column the chief executive of the NHS Confederation, Rob Webster, seeks to cut through the political row and conflicting messages to spell out the things that are needed from the government financially to support the NHS in the next parliament (doi:10. 1136/bmj.h1978). "It's time to bring clarity on what is needed in the NHS," writes Webster. "The money is one challenge, transforming care is an even greater one. If we had all the money in the world we would still need to change the NHS."

For a possible model of how to transform care we could look to Basildon and Thurrock University Hospitals NHS Foundation Trust. As Matthew Limb reports (doi:10.1136/bmj.h1976), in July 2013 it was one of 11 NHS foundation trusts put into special measures because of high death rates. Within a year, and after many new initiatives, including a boost to the trust's clinical leadership, it was rated "good" overall by the Care Quality Commission. Its associate medical director Charlotte Hopkins says that now the focus is "very much on how you go from good to outstanding."

This week's contributor to our If I Ruled the NHS series has clear ideas about how to transform care (doi:10.1136/bmj. h1759). Chris Holcombe, a consultant breast surgeon from Liverpool, would ensure that every candidate for every interview for every NHS post-whether clinical or managerial and administrative - was asked how much they care. "Care, what it is, how it is practically applied, and how it could be improved would be discussed and considered thousands of times every day," he writes. "Care would become everyone's concern."

Holcombe believes that this strategy might help prevent a climate in which so many of the stories in the news are about failure, and particularly failure in care. Editorialists Hanan Edrees and Frank Federico focus on the "second victims" of medical error or adverse events in healthcare: the clinicians involved, who may be "suffering in silence" (doi:10.1136/bmj. h1982). "These healthcare providers are often told to take care of the next patient without an opportunity to discuss the details of the event or share how this has affected them personally and professionally," they write. Edrees and Federico call for the development of more structured support programmes to help the "second victims."

In her weekly column the general practitioner Margaret McCartney admits that every day she fails to do things as well as she should (doi:10.1136/bmj.h1331). "I don't spend enough time discussing this preventive strategy or that treatment. I don't spend enough time talking about what drugs we should stop or why an alternative treatment is wasting money." Reflecting on the "true" meaning of partnership with patients, McCartney fears that the target culture means that doctors are stuck working for the government rather than patients, and she, too, wants to be emancipated. "We need a revolution where patients truly come first, not politicians."

For a clearer picture of what the priorities are for politicians, Gareth Iacobucci has summarised the NHS related proposals from the parties' manifestos (doi:10.1136/bmj.h2031). Tell us what you think of the proposals in a rapid response.

The $B M J$ is co-hosting a UK general election hustings with the health spokespeople from the main political parties at $11 \mathrm{am}$ on 21 April. Watch the debate live at www.healthdebate.net

For more from The BMJ on the UK general election go to bmj.co/election.

Cite this as: BMJ 2015;350:h2027

๑ BMJ Publishing Group Ltd 2015 